\title{
Clinical characteristics and causes of heart failure, adherence to treatment guidelines, and mortality of patients with acute heart failure: Experience at Groote Schuur Hospital, Cape Town, South Africa
}

\author{
P Z Szymanski, ${ }^{1} \mathrm{MB} \mathrm{ChB} ; \mathbf{M}$ Badri, ${ }^{2} \mathrm{PhD} ; \mathbf{B}$ M Mayosi, ${ }^{1} \mathrm{MB} \mathrm{ChB}, \mathrm{DPhil}$ \\ ${ }^{1}$ Department of Medicine, Groote Schuur Hospital and Faculty of Health Sciences, University of Cape Town, South Africa \\ ${ }^{2}$ Department of Medicine, Cardiac Clinic, Groote Schuur Hospital and Faculty of Health Sciences, University of Cape Town, South Africa
}

Corresponding author: P Z Szymanski(dr.patryk@gmail.com)

Background. There is limited information on acute heart failure (AHF) and its treatment in sub-Saharan Africa.

Objective. To describe the clinical characteristics and causes of heart failure (HF), adherence to HF treatment guidelines, and mortality of patients with AHF presenting to Groote Schuur Hospital (GSH), Cape Town, South Africa.

Methods. This sub-study of The Sub-Saharan Africa Survey of Heart Failure (THESUS-HF) was a prospective and observational survey that focused on the enrolment and follow-up of additional patients with AHF presenting to GSH and entered into the existing registry after publication of the primary THESUS-HF article in 2012. The patients were classified into prevalent (existing) or incident (new) cases of HF. Results. Of the 119 patients included, 69 (58.0\%) were female and the mean (standard deviation) age was 49.9 (16.3) years. The majority of prevalent cases were patients of mixed ancestry (63.3\%), and prevalent cases had more hypertension (70.0\%), diabetes mellitus (36.7\%), hyperlipidaemia (33.3\%) and ischaemic heart disease (IHD) (36.7\%) than incident cases. The top five causes of HF were cardiomyopathy (20.2\%), IHD (19.3\%), rheumatic valvular heart disease (RHD) (18.5\%), cor pulmonale (11.8\%) and hypertension (10.1\%), with the remaining $20.1 \%$ consisting of miscellaneous causes including pericarditis, toxins and congenital heart disease. Most patients received renin-angiotensin system blockers and loop diuretics on discharge. There was a low rate of beta-blocker, aldosterone antagonist and digoxin use. Rehospitalisation within 180 days occurred in $25.2 \%$ of cases. In-hospital mortality was $8.4 \%$ and the case fatality rate at 6 months was $26.1 \%$.

Conclusion. In Cape Town, the main causes of AHF are cardiomyopathy, IHD and RHD. AHF affects a young population and is associated with a high rate of rehospitalisation and mortality. There is serious under-use of beta-blockers, aldosterone antagonists and digoxin. Emphasis on the rigorous application of treatment guidelines is needed to reduce readmission and mortality.

S Afr Med J 2018;108(2):94-98. DOI:10.7196/SAMJ.2018.v108i2.12519

Heart failure (HF) is a worldwide phenomenon that affects millions of people yearly and carries a high mortality. It is estimated that 37.7 million people worldwide are affected by HF ${ }^{[1]}$ The past three decades have seen a rise in research on HF. Large multicentre studies from the USA (e.g. ADHERE) and Europe (e.g. EHFS II) have provided greater insight into the aetiology, treatment and outcomes of patients with $\mathrm{HF}$ in the developed world. ${ }^{[2,3]}$

Observational studies from sub-Saharan Africa (SSA) show that hypertension, rheumatic valvular heart disease (RHD) and idiopathic cardiomyopathies are the main causes of HF affecting a young population. ${ }^{[4-6]}$ This epidemiological pattern is strikingly different from that in the developed world, where a much older population suffers from HF, with ischaemic heart disease (IHD) the primary cause. ${ }^{[2,3]}$ Despite these differences, the epidemiological transition is resulting in the rise of diabetes mellitus (DM), hypertension and IHD in SSA. ${ }^{[7,8]}$ In 2008, communicable, maternal, perinatal and nutritional conditions accounted for the majority of deaths in South Africa (SA). However, of the non-communicable diseases, cardiovascular disease was the main cause of death. ${ }^{[9]}$

There is limited information on the use of evidence-based interventions and outcomes of HF in Africa. Contemporary epidemiological, aetiological and treatment information is needed in order to develop appropriate health policies for its diagnosis, management, prevention and control. ${ }^{[10,11]}$

\section{Objective}

To explore the treatment practices of doctors and outcomes of patients with congestive or acute heart failure (AHF) at Groote Schuur Hospital (GSH), a tertiary-level academic institution in Cape Town, SA, that serves as a referral hospital for 5 million people. The clinical features and causes of HF were also explored.

\section{Methods}

Study design and clinical setting

This was a sub-study of The Sub-Saharan Africa Survey of Heart Failure (THESUS-HF), a prospective, multicentre, observational survey of patients with AHF admitted to 12 university hospitals in nine countries. ${ }^{[5]}$ The sub-study reported here is based on the enrolment of additional patients with AHF presenting to GSH and followed up in the existing THESUS-HF registry. Patients with AHF (incident (i.e. de novo) or prevalent (i.e. decompensation of previously diagnosed $\mathrm{HF}$ )) were added to the registry after the publication of the primary THESUS-HF article in 2012. ${ }^{[5]}$

\section{Inclusion and exclusion criteria}

The inclusion and exclusion criteria were the same as the criteria in the THESUS-HF study ${ }^{[5]}$ Briefly, patients aged $>12$ years with a diagnosis of AHF based on clinical evaluation and confirmed by echocardiography were enrolled. The exclusion criteria were 
acute ST-elevation myocardial infarction, known severe renal failure (patients undergoing dialysis or with a creatinine level $>350 \mu \mathrm{mol} / \mathrm{L}$ ), nephrotic syndrome, hepatic failure or other causes of hypoalbuminaemia.

\section{Case definition and data collection}

The diagnosis of HF was based on the standard case definition as described in THESUS-HF. ${ }^{[5]}$ The diagnosis of HF was made on finding the clinical syndrome of effort intolerance (i.e. shortness of breath, dyspnoea and/or fatigue) associated with features of fluid retention (i.e. peripheral oedema, orthopnoea, paroxysmal nocturnal dyspnoea, raised jugular venous pressure, pulmonary oedema and/ or tender hepatomegaly) in the presence of clinical signs of cardiac dysfunction (i.e. low blood pressure, displaced apex, presence of third heart sound). The presence of cardiac dysfunction was confirmed by echocardiography performed by a trained echocardiographer. The cause of HF was based on information obtained from the history, physical examination, echocardiography, and special aetiological investigations interpreted by the admitting team on the index admission. Where no cause for HF was stated, the research team determined the aetiology from the given data.

For this study, consecutive patients enrolled into the GSH THESUS-HF registry from 1 June 2012 to 31 May 2014 (24 months) with $>90 \%$ completed data were selected. The THESUS-HF registry holds the basic clinical information as determined by the clinical history and previous medical records, along with an echocardiogram report confirming the diagnosis of HF. Furthermore, patients were followed up for 180 days to document their clinical outcomes of rehospitalisation and death. Their baseline demographic and clinical information, echocardiographic findings, treatment and clinical outcomes were entered onto a data capture sheet by the research nurse and echocardiographer. Outstanding demographic, clinical, treatment and outcomes data were collected through folder review, pharmacy records and telephonic consultations with the study participants. All completed data capture sheets were entered into the THESUS-HF database.

\section{Statistical analysis}

Data were analysed using the Statistical Package for the Social Sciences (SPSS) version 23 (IBM, USA) and ROOT. ${ }^{[12]}$ Normally distributed continuous data are presented as means with standard deviations (SDs), and non-Gaussian distributed variables as medians plus ranges. Categorical data are presented as percentages. The case fatality rate was calculated at discharge and at 6 months of follow-up.

\section{Ethical considerations}

The University of Cape Town Human Research Ethics Committee granted ethics approval for this study (ref. no. 579/2014). All patients entered into the THESUS-HF registry gave written informed consent.

\section{Results}

\section{Baseline patient characteristics on admission}

One hundred and twenty patients were enrolled in the THESUS-HF registry at GSH between 1 June 2012 and 31 May 2014. One patient was excluded owing to missing clinical details. Table 1 shows the baseline clinical characteristics for the total cohort $(N=119)$ and compares the incident cases (first presentations) with the prevalent cases (recurrent presentations). The mean (SD) age of the cohort was 49.9 (16.3) years. Sixty-nine patients $(57.5 \%)$ were female, and the main population groups were black African $(n=59,49.6 \%)$ and mixed ancestry $(n=54,45.4 \%)$.
The prevalent cases were more likely to be people of mixed ancestry, and this group had more hypertension, DM, hyperlipidaemia, IHD, pericardial disease and cardiomyopathy and higher New York Heart Association (NYHA) functional class than incident cases.

\section{Causes of HF}

The main causes of HF were cardiomyopathy $(n=24,20.2 \%)$, IHD $(n=23,19.3 \%)$ and valvular heart disease $(n=22,18.5 \%)$. Hypertension accounted for $10.1 \%(n=12)$ of the cohort. Owing to the small sample size, cardiomyopathy represents the cumulative causes of cardiomyopathy, and includes peripartum cardiomyopathy, idiopathic dilated cardiomyopathy and HIV cardiomyopathy. Fig. 1 shows the causes of HF.

\section{Therapies for HF: Admission and discharge/day 7 admission}

Intravenous (IV) loop diuretics were the most commonly used IV therapy on admission ( $n=94,79.0 \%)$, while renin-angiotensin system blockers were the most commonly used oral treatment $(n=70,59.3 \%)$. Mechanical ventilation and dobutamine were used in only $0.9 \%(n=1)$ and $1.7 \%(n=2)$ of cases, respectively. Reninangiotensin system blockers $(73.0 \%, n=81)$, loop diuretics $(74.6 \%$, $n=82)$ and beta-blockers $(42.7 \%, n=47)$ were most commonly issued on discharge. On discharge only $26.1 \%$ of patients $(n=15)$ received aldosterone antagonists and $15.5 \%(n=17)$ digoxin. IV dopamine, IV digoxin and oral hydralazine were never prescribed. Table 2 shows the IV and oral therapies used in incident and prevalent cases.

\section{Length of stay, rehospitalisation and case fatality rate}

The mean (SD) length of stay in hospital for all cases was 9.2 (12.2) days, with a median of 6 days (range 1 - 109). Rehospitalisation within 180 days occurred in $25.2 \%$ ( $n=30$ ) of the total cohort. Twelve patients (10.1\%) were lost to follow-up. The main reason for this was no reply to telephonic calls $(n=11)$, and one patient moved to another province. The rate of death during hospital admission was $8.4 \%(10 / 119$ patients), and the case fatality rate at 6 months was $26.1 \%$ (31/119 patients).

\section{Discussion}

The main findings of this study include a relatively young population suffering from HF, with prevalent cases more likely to have DM, hypertension, IHD and more advanced disease. Furthermore, cardiomyopathy, IHD and RHD account for the majority of causes of HF. There was under-use of beta-blockers, aldosterone antagonists and digoxin. Finally, rehospitalisation and case fatality rates were high.

The mean age of patients with AHF was 49.9 years, and $58.0 \%$ were female. These findings are similar to what has been observed in other registries from SSA. ${ }^{[4,5,13,14]}$ This is significant, as it demonstrates that HF in Cape Town affects the breadwinner generation, rather than the elderly as has been noted in the developed world. In contrast, the average age of patients with AHF in Europe and North America is 70 years. ${ }^{[2,3]}$

There was also a difference in clinical characteristics between patients with new-onset disease and those with a previous diagnosis of HF. The patients with prevalent disease were mostly from the mixed-ancestry community. This may be representative of Cape Town's population demographics, the mixed-ancestry community constituting the largest group in the Western Cape. ${ }^{[15]}$ Furthermore, patients with prevalent disease had more hypertension, DM, hyperlipidaemia and IHD. Comparison of coronary artery disease 
Table 1. Baseline clinical characteristics of 119 patients with AHF at GSH, Cape Town, SA

\begin{tabular}{|c|c|c|c|c|}
\hline Characteristics & All cases $(N=119)$ & Incident cases $(N=89)$ & Prevalent cases $(N=30)$ & $p$-value \\
\hline Age (yr), mean (SD) & $49.9(16.3)$ & $48.6(16.2)$ & $53.7(16.4)$ & 0.143 \\
\hline Sex, $n(\%)$ & & & & 0.349 \\
\hline Female & $69(58.0)$ & $53(59.6)$ & $16(53.3)$ & \\
\hline Male & $50(42.0)$ & $36(40.4)$ & $14(46.7)$ & \\
\hline Race, $n(\%)$ & & & & 0.005 \\
\hline Black & $59(49.6)$ & $51(57.3)$ & $8(26.7)$ & \\
\hline White & $4(3.4)$ & $3(3.4)$ & $1(3.3)$ & \\
\hline Mixed ancestry & $54(45.4)$ & $35(39.3)$ & $19(63.3)$ & \\
\hline Asian & $2(1.7)$ & 0 & $2(6.7)$ & \\
\hline AHF admissions in past 12 months, $n(\%)$ & & & & $\mathrm{n} / \mathrm{a}$ \\
\hline 0 & $89(74.8)$ & $89(100)$ & & \\
\hline 1 & $22(18.5)$ & & $22(73.3)$ & \\
\hline 2 & $4(3.4)$ & & $4(13.3)$ & \\
\hline 3 & $4(3.4)$ & & $4(13.3)$ & \\
\hline Hypertension, $n(\%)$ & $58(48.7)$ & $37(41.6)$ & $21(70.0)$ & 0.006 \\
\hline $\mathrm{DM}, n(\%)$ & $26(21.8)$ & $15(16.9)$ & $11(36.7)$ & 0.025 \\
\hline Smoking, $n(\%)$ & $46(38.7)$ & $31(34.8)$ & $15(50)$ & 0.105 \\
\hline Hyperlipidaemia, $n$ (\%) & $13(10.9)$ & $3(3.4)$ & $10(33.3)$ & $<0.001$ \\
\hline $\mathrm{IHD}, n(\%)$ & $17(14.3)$ & $6(6.7)$ & $11(36.7)$ & $<0.001$ \\
\hline Atrial fibrillation, $n(\%)$ & $6(5.0)$ & $5(5.6)$ & $1(3.3)$ & 0.526 \\
\hline Stroke, $n(\%)$ & $5(4.2)$ & $3(3.4)$ & $2(6.7)$ & 0.372 \\
\hline Pericardial disease, $n(\%)$ & $3(2.5)$ & 0 & $3(10.0)$ & 0.015 \\
\hline RHD, $n(\%)$ & $12(10.1)$ & $10(11.2)$ & $2(6.7)$ & 0.374 \\
\hline Cardiomyopathy, $n$ (\%) & $11(9.2)$ & $4(4.5)$ & $7(23.3)$ & 0.005 \\
\hline Cor pulmonale, $n(\%)$ & $7(5.9)$ & $3(3.4)$ & $4(13.3)$ & 0.066 \\
\hline HIV, $n(\%)$ & $14(11.8)$ & $10(11.2)$ & $4(13.3)$ & 0.490 \\
\hline PVD, $n(\%)$ & $2(1.7)$ & $2(2.2)$ & 0 & 0.558 \\
\hline NYHA, $n(\%)$ & & & & $<0.001$ \\
\hline $\mathrm{I}$ & $23(23.7)$ & $23(34.3)$ & 0 & \\
\hline II & $20(20.6)$ & $17(25.4)$ & $3(10.0)$ & \\
\hline III & $44(45.4)$ & $24(35.8)$ & $20(66.7)$ & \\
\hline IV & $10(10.3)$ & $3(4.5)$ & $7(23.3)$ & \\
\hline BMI $\left(\mathrm{kg} / \mathrm{m}^{2}\right)$, mean (SD) & $27.4(9.8)$ & $27.6(10.5)$ & $26.7(7.6)$ & 0.680 \\
\hline Systolic BP (mmHg), mean (SD) & $134.6(33.2)$ & $137.4(32.9)$ & $126.3(33.3)$ & 0.115 \\
\hline Diastolic BP (mmHg), mean (SD) & $81.9(22.5)$ & $82.4(23.3)$ & $80.5(20.1)$ & 0.688 \\
\hline Heart rate (bpm), mean (SD) & $102.5(22.7)$ & $102.7(24.3)$ & $101.9(17.3)$ & 0.865 \\
\hline Respiratory rate (/min), mean (SD) & $22.8(5.3)$ & $23.0(5.7)$ & $22.0(3.5)$ & 0.370 \\
\hline Oedema, $n(\%)$ & & & & 0.762 \\
\hline 0 & $37(31.1)$ & $27(30.3)$ & $10(33.3)$ & \\
\hline $1+$ & $1(0.8)$ & $1(1.1)$ & 0 & \\
\hline $2+$ & $21(17.6)$ & $17(19.1)$ & $4(13.3)$ & \\
\hline $3+$ & $60(50.4)$ & $44(49.4)$ & $16(53.3)$ & \\
\hline Pulmonary oedema, $n(\%)$ & & & & 0.038 \\
\hline 0 & $46(38.7)$ & $33(37.1)$ & $13(43.3)$ & \\
\hline 1 & $4(3.4)$ & $2(2.2)$ & $2(6.7)$ & \\
\hline 2 & $28(23.5)$ & $26(29.2)$ & $2(6.7)$ & \\
\hline 3 & $41(34.5)$ & $28(31.5)$ & $13(43.3)$ & \\
\hline LVEF (\%), mean (SD) & $34.1(16.9)$ & $35.9(17.4)$ & $28.2(14.0)$ & 0.085 \\
\hline Creatinine $(\mu \mathrm{mol} / \mathrm{dL})$, mean $(\mathrm{SD})$ & $109.7(75.4)$ & $110.2(83.9)$ & $108.4(43.5)$ & 0.912 \\
\hline Urea $(\mathrm{mmol} / \mathrm{L})$, mean $(\mathrm{SD})$ & $10.2(7.5)$ & $9.7(7.2)$ & $11.6(8.3)$ & 0.250 \\
\hline Sodium (mmol/L), mean (SD) & $136.9(6.3)$ & $137.0(6.6)$ & $136.9(5.7)$ & 0.943 \\
\hline Haemoglobin (g/dL), mean (SD) & $12.3(2.6)$ & $12.1(2.7)$ & $12.7(2.4)$ & 0.329 \\
\hline White cell count $\left(\times 10^{9} / \mathrm{L}\right)$, mean $(\mathrm{SD})$ & $9.7(4.5)$ & $9.8(4.5)$ & $9.2(4.5)$ & 0.482 \\
\hline \multicolumn{5}{|c|}{ 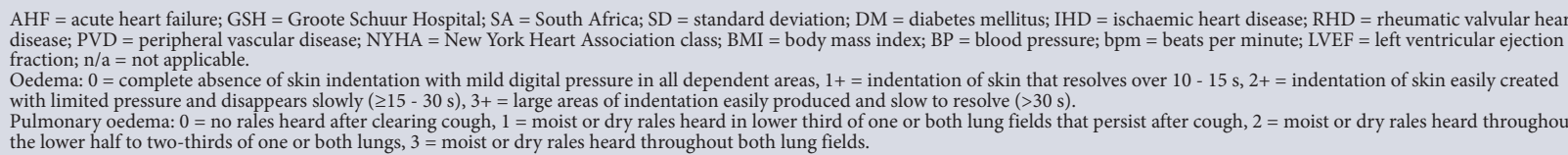 } \\
\hline
\end{tabular}


risk factors in the present study with those of other HF studies in SSA shows a greater burden of DM and smoking (Table 3). Finally, their baseline NYHA functional class was higher, possibly indicating a group of patients with more advanced HF, which may reflect suboptimal treatment of HF along with poor follow-up and education regarding HF.

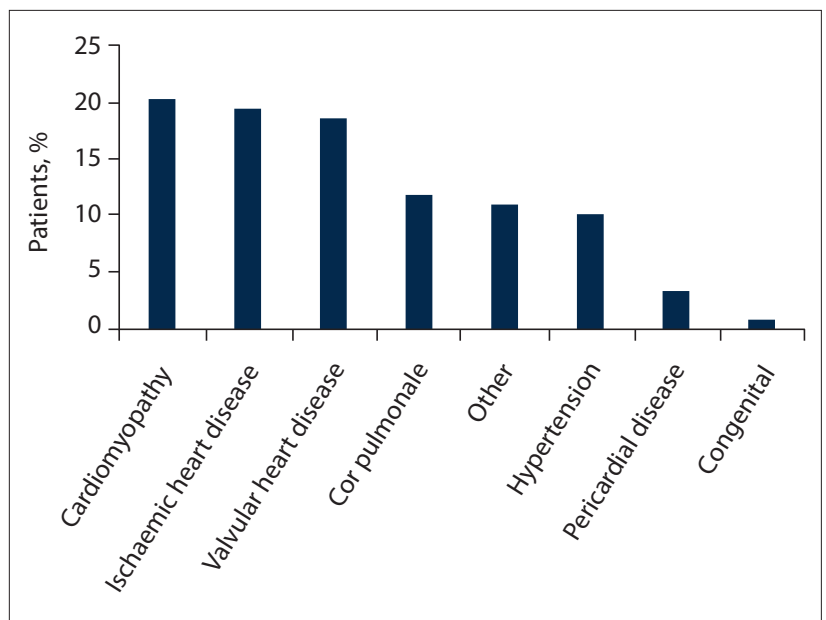

Fig. 1. Causes of heart failure. ('Other' includes toxins, arrhythmias and Graves' disease.)
In SSA, hypertension, RHD and the endemic cardiomyopathies account for the majority of HF cases. ${ }^{[5]}$ In this study, cardiomyopathy, IHD and RHD were the leading causes of AHF in Cape Town, accounting for $60 \%$ of cases referred to tertiary care. Of interest is the high prevalence of IHD. Observational studies from SSA report the incidence of IHD as a cause of HF to be $0.4-9 \%{ }^{[4,5,13,14]}$ In this study, $19.3 \%$ of patients were diagnosed with an ischaemic cause of HF. One of the possible reasons for the disparity in observations may be the effects of urbanisation. It has been shown that movement of people from rural areas to urban centres results in a change in diet and lifestyle, predisposing to the development of IHD. ${ }^{[7,8]}$ Another reason may be that a specialist cardiology service was available to this study cohort, which may have allowed for better diagnostic tests (angiography and echocardiography), resulting in increased diagnosis of IHD. Hypertensive HF was less prevalent in this study than in other series from SSA. The reason for this is not clear, and requires further investigation.

The rate of beta-blocker use was low at discharge in this study (42.7\%) compared with EHFS II (61\%), but was similar to rates of use observed in the Tanzania Heart Failure (TaHeF) study (42\%) and THESUS-HF (50\%). ${ }^{[3,5,14]}$ The low rate of beta-blocker use may be due to the severity of HF treated. In this cohort, over half of the patients presented with NYHA functional classes III and IV combined. On discharge, $26.1 \%$ of patients received aldosterone antagonists and $15.5 \%$ received digoxin. This is considerably lower than has been

Table 2. Prescribed medication on admission and discharge or day 7 admission

\begin{tabular}{|c|c|c|c|c|c|c|}
\hline \multirow[b]{2}{*}{ Treatment } & \multicolumn{2}{|c|}{ All cases } & \multicolumn{2}{|c|}{ Incident cases } & \multicolumn{2}{|c|}{ Prevalent cases } \\
\hline & Admission, $n(\%)$ & $\begin{array}{l}\text { Discharge/d 7, } \\
n(\%)\end{array}$ & Admission, $n$ (\%) & $\begin{array}{l}\text { Discharge/d 7, } \\
n(\%)\end{array}$ & Admission, $n$ (\%) & $\begin{array}{l}\text { Discharge/d 7, } \\
n(\%)\end{array}$ \\
\hline Nitrates (IV) & $4(3.5)$ & 0 & $3(3.5)$ & 0 & $1(3.3)$ & 0 \\
\hline Furosemide (IV) & $94(79.0)$ & $20(18.0)$ & $71(79.8)$ & $13(15.9)$ & $23(76.7)$ & $7(24.1)$ \\
\hline Dobutamine & $2(1.7)$ & 0 & $2(2.4)$ & 0 & 0 & 0 \\
\hline Mechanical ventilation & $1(0.9)$ & 0 & 0 & 0 & $1(3.3)$ & 0 \\
\hline ACE inhibitor/ARB & $70(59.3)$ & $81(73.0)$ & $48(54.5)$ & $57(69.5)$ & $22(73.3)$ & $24(82.8)$ \\
\hline Loop diuretics & $24(20.7)$ & $82(74.6)$ & $17(19.8)$ & $60(74.1)$ & $7(23.3)$ & $22(75.9)$ \\
\hline Beta-blockers & $21(18.3)$ & $47(42.7)$ & $13(15.3)$ & $32(39.5)$ & $8(26.7)$ & $15(51.7)$ \\
\hline Digoxin & $15(13.2)$ & $17(15.5)$ & $9(10.6)$ & $12(14.8)$ & $6(20.7)$ & $5(17.2)$ \\
\hline Nitrates & $4(3.5)$ & $9(8.2)$ & $3(3.5)$ & $4(4.9)$ & $1(3.3)$ & $5(17.2)$ \\
\hline $\begin{array}{l}\text { Aldosterone } \\
\text { antagonists }\end{array}$ & 15 (12.9) & $29(26.1)$ & $9(10.5)$ & $19(23.2)$ & $6(20.0)$ & $10(34.5)$ \\
\hline Simvastatin & $36(30.8)$ & $36(32.4)$ & $21(24.1)$ & $21(25.6)$ & $15(50.0)$ & $15(51.7)$ \\
\hline Aspirin & $30(25.6)$ & $32(28.8)$ & 19 (21.8) & $19(23.2)$ & $11(36.7)$ & $13(44.8)$ \\
\hline All anticoagulation ${ }^{*}$ & $45(38.5)$ & $32(28.8)$ & $32(36.8)$ & $26(31.7)$ & $13(43.3)$ & $6(20.7)$ \\
\hline
\end{tabular}

Table 3. Comparison of CAD risk factors in the present study with HF studies from SSA and other parts of the world

\begin{tabular}{llll}
\hline Study, country & Diabetes (\%) & Hypertension (\%) & Smoking (\%) \\
\hline Current study & 21.8 & 48.7 & 38.7 \\
Stewart et al..,$^{[4]}$ SA & 10 & 55 & 48 \\
Lokker et al. ${ }^{[18]}$ SA & 24 & 70 & NR \\
Mwita et al..$^{[1]]}$ Botswana & 15.5 & 54.9 & NR \\
Ogah et al..${ }^{[13]}$ Nigeria & 10 & 64.3 & 3.3 \\
Makubi et al...$^{[14]}$ Tanzania & 12 & 45 & NR \\
THESUS-HF ${ }^{[5]}$ & 11.1 & 55 & 9.8 \\
EHFS II, ${ }^{[3]}$ Europe & 62.5 & 32.8 & NR \\
ADHERE, ${ }^{[2]}$ USA & 44 & 73 & NR
\end{tabular}

$\mathrm{CAD}=$ coronary artery disease; $\mathrm{HF}=$ heart failure; $\mathrm{SSA}=$ sub-Saharan Africa; $\mathrm{SA}=$ South Africa; THESUS-HF $=$ The Sub-Saharan Africa Survey of Heart Failure; $\mathrm{EFHS} \mathrm{II} \mathrm{=} \mathrm{EuroHeart} \mathrm{Failure}$ Survey II; ADHERE = Acute Decompensated Heart Failure National Registry; CAD = Coronary Artery Disease; NR = not reported. 
described in the rest of SSA, where aldosterone and digoxin are prescribed in $60-75 \%$ and $31-72 \%$ of cases, respectively. ${ }^{[4,5,13,14]}$ The side-effect profiles, potential drug interactions and close monitoring of these therapies in a population group where compliance to follow-up and access to specialist care is suboptimal may explain their limited use. Hydralazine use was low, possibly because of its limited availability at GSH and also its known low use by physicians in SSA. ${ }^{[5]}$ In this study, $28.8 \%$ of patients were discharged on aspirin. This is a far lower figure than is described in THESUS-HF, where $>50 \%$ of patients were discharged on aspirin despite the low prevalence of ischaemic HF. ${ }^{[5]}$ This study shows a population with a high prevalence of IHD. Whether the use of aspirin in this study is in keeping with the prevalence of IHD as identified in this cohort needs to be evaluated further.

The mean length of stay of 9.2 days is similar to the number of admission days described in studies from developed nations and SSA. ${ }^{[3-5,13]}$ The 6 -month readmission rate was $25.2 \%$. The reasons for this high readmission rate may be three-fold. First, suboptimal adherence to evidence-based treatments may be resulting in high readmission rates. Second, gaps in patients' understanding of HF and the importance of treatment follow-up and lifestyle adherence may be a further contributory factor ${ }^{[16]}$ Finally, hospital bed pressure may lead to premature discharge of HF patients. The in-hospital and 6 -month mortality rates were $8.4 \%$ and $26.1 \%$, respectively. This is higher than is described in the rest of SSA. THESUS-HF, the largest multicentre study on HF to date, reported an in-hospital mortality rate of $4.2 \%$ and a 6 -month mortality rate of $17.8 \%{ }^{[5]}$ These high mortality rates may be due to the different aetiological pattern in Cape Town, with a significant contribution of IHD and a lower prevalence of hypertension as the primary cause of AHF. There was also low use of life-saving medications such as beta-blockers and aldosterone inhibitors. These observations stress the importance of adhering to evidence-based treatment guidelines. Furthermore, the management of HF is complex and requires a multidisciplinary approach encompassing routine follow-up with patient education, optimisation of treatment and social support. ${ }^{[17]}$

\section{Study limitations}

This study has a number of limitations. The sample size was small and may under-represent the causes of HF. A second limitation was that rehospitalisation to other institutions may not have been documented in all cases. Furthermore, only admission and discharge treatment practices are described. It may be that the use of aldosterone and beta-blockers as well as other HF treatment increased during ambulatory care in the community. There is also lack of data on dosages of drugs that confer survival benefit in HF. Finally, $10.1 \%$ of the patient cohort was lost to follow-up, which may have contributed to under-estimation of the case fatality rate.

\section{Conclusion}

This study provides important insights into the demographics, causes, treatment and outcomes of AHF in Cape Town. It confirms observations in earlier SSA HF studies that a young adult population is affected by HF. Cardiomyopathy, IHD and RHD account for $60 \%$ of cases of AHF. However, of concern is the high prevalence of IHD in this cohort, the highest reported so far in SSA. Furthermore, there was serious under-use of beta-blockers, aldosterone antagonists and digoxin. The high readmission rate and 6-month mortality rate may reflect suboptimal adherence to evidence-based treatment guidelines. There needs to be emphasis on the rigorous application of treatment guidelines to reduce readmission and mortality associated with AHF in Cape Town.

Acknowledgements. We thank Sisters Dee Isaacs and Veronica Francis for help with the recruitment of patients and collection of data for this study, and Mr Lwazi Mhlanti for the development of the database and data capturing. We also acknowledge the THESUS-HF Consortium (Drs G Cotter, A Damasceno and K Sliwa) for permission to conduct the substudy in Cape Town.

Author contributions. PZS drafted the manuscript and managed the data collection. MB performed the data analysis and assisted with data preparation for statistical analysis. BMM supervised the study and was a major contributor to the study design and final manuscript.

Funding. The study was funded by the Discovery Foundation (through an MMed Fellowship to PZS), the Lily and Ernst Hausmann Research Trust, the South African Medical Research Council and AstraZeneca.

Conflicts of interest. None.

1. Ziaeian B, Fonarow GC. Epidemiology and aetiology of heart failure. Nat Rev Cardiol 2016;13(6):368378. https://doi.org/10.1038/nrcardio.2016.25

2. Adams KF, Fonarow GC, Emerman CL, et al. Characteristics and outcomes of patients hospitalized for heart failure in the United States: Rationale, design, and preliminary observations from the first
forstitis for heart failure in the United States: Rationale, design, and preliminary observations from the first
100,000 cases in the Acute Decompensated Heart Failure National Registry (ADHERE). Am Heart J 100,000 cases in the Acute Decompensated Heart Failure Nation
2005;149(2):209-216. https://doi.org/10.1016/j.ahj.2004.08.005

3. Nieminen MS, Brutsaert D, Dickstein K, et al. EuroHeart Failure Survey II (EHFS II): A survey on
(2) hospitalized acute heart failure patients: Description of population. Eur Heart J 2006;27(22):2725-2736. https://doi.org/10.1093/eurheartj/ehl193

4. Stewart S, Wilkinson D, Hansen C, et al. Predominance of heart failure in the Heart of Soweto Stud cohort: Emerging challenges for urban African communities. Circulation 2008;118(23):2360-2367 https://doi.org/10.1161/CIRCULATIONAHA.108.786244

5. Damasceno A, Mayosi BM, Sani M, et al. The causes, treatment, and outcome of acute heart failure in 1006 Africans from 9 countries. Arch Intern Med 2012;172(18):1386-1394. https://doi.org/10.1001/ archinternmed.2012.3310

6. Ntusi NBA, Mayosi BM. Epidemiology of heart failure in sub-Saharan Africa. Expert Rev Cardiovasc Ther 2009; 7(2):169-180. https://doi.org/10.1586/14779072.7.2.169

7. Mendez GF, Cowie MR. The epidemiological features of heart failure in developing countries: A review of Mendez GF, Cowie MR. The epidemiological features of heart failure in developing countries: A re
the literature. Int J Cardiol 2001;80(2-3):213-219. https://doi.org/10.1016/50167-5273(01)00497-1

the literature. Int J Cardiol 2001;80(2-3):213-219. https://doi.org/10.1016/s0167-5273(01)00497-1
8. Stewart S, Carrington M, Pretorius S, Methusi P, Sliwa K. Standing at the crossroads between new and Stewart S, Carrington M, Pretorius S, Methusi P, Sliwa K. Standing at the crossroads between new and
historically prevalent heart disease: Effects of migration and socio-economic factors in the Heart of Soweto cohort study. Eur Heart J 2011;32(4):492-499. https://doi.org/10.1093/eurheart//ehq439

9. Alwan A, Armstrong T, Cowan M, Riley L. Noncommunicable Diseases Country Profiles 2011. World Health Organization, 2011:1-207. http://www.who.int/nmh/publications/ncd_profiles2011/en/ (accessed 2 January 2018).

0. Commerford P, Mayosi B. An appropriate research agenda for heart disease in Africa. Lancet 2006;367(9526):1884-1886. https://doi.org/10.1016/\$0140-6736(06)68822-3

11. Sliwa K, Mayosi BM. Recent advances in the epidemiology, pathogenesis and prognosis of acute heart failure and cardiomyopathy in Africa. Heart 2013;99(18):1317-1322. https://doi.org/10.1136/ heartjnl-2013-303592

12. Brun R. ROOT - an object oriented data analysis framework. Nucl Instrum Methods Phys Res A 1997;389(1-2):81-86. https://doi.org/10.1016/S0168-9002(97)00048-X

13. Ogah OS, Stewart S, Falase AO, et al. Contemporary profile of acute heart failure in Southern Nigeria: Data from the Abeokuta Heart Failure Clinical Registry. JACC Heart Fail 2014;2(3):250-259. https://doi. Data from the Abeokuta Heart
org/10.1016/j.jchf.2013.12.005

14. Makubi A, Hage C, Lwakatare J, et al. Contemporary aetiology, clinical characteristics and prognosis Makubi A, Hage C, Lwakatare J, et al. Contemporary aetiology, clinical characteristics and prognosis
of adults with heart failure observed in a tertiary hospital in Tanzania: The prospective Tanzania Heart Failure (TaHeF) study. Heart 2014;100(16):1235-1241. https://doi.org/10.1136/heartjnl-2014-305599

15. Statistics South Africa. Census 2011: Census in Brief. Pretoria: Stats SA, 2012. http://www.statssa.gov. za/census/census_2011/census_products/Census_2011_Census_in_brief.pdf (accessed 2 January 2018). 16. Van der Wal MHL, Jaarsma T, van Veldhuisen DJ. Non-compliance in patients with heart failure: How can we manage it? Eur J Heart Fail 2005;7(1):5-17. https://doi.org/10.1016/j.ejheart.2004.04.007

17. Mpe MT, Klug EQ, Sliwa KS, Hitzeroth J, Smith DA. Heart Failure Society of South Africa (HeFSSA perspective on the European Society of Cardiology (ESC) 2012 chronic heart failure guideline. S Afr Med J 2013;103(9):661-667. https://doi.org/10.7196/SAMJ.7319

18. Lokker ME, Gwyther L, Riley JP, van Zuylen L, van der Heide A, Harding R. The prevalence and associated distress of physical and psychological symptoms in patients with advanced heart failure attending a South African medical center. J Cardiovasc Nurs 2016;31(4):313-322. https://doi.org/10.1097/ JCN.0000000000000256

19. Mwita JC, Dewhurst MJ, Magafu MG, et al. Presentation and mortality of patients hospitalised with acute heart failure in Botswana. Cardiovasc J Afr 2016;28(2):112-117. https://doi.org/10.5830/CVJA-2016-067 